\title{
Teaching Supervisory Control Based on a Web Portal and a System of Laboratory Tasks
}

\author{
Lenka Landryová ${ }^{1}$, Iveta Zolotová ${ }^{2}$, and Marián Bakoš ${ }^{2}$ \\ 1 Department of Control Systems and Instrumentation, Faculty of \\ Mechanical Engineering, VŠB - Technical University Ostrava, \\ 17. listopadu 15, 70833 Ostrava-Poruba, Czech Republic, \\ Lenka.Landryova@vsb.cz \\ 2 Department of Cybernetics and Artificial Intelligence, Faculty of Electro \\ Engineering and Informatics, Technical University Košice, Letná 9, 04200 \\ Košice, Slovak Republic \\ \{Iveta.Zolotova, Marian.Bakos\}@tuke.sk
}

\begin{abstract}
This paper was written based on experience with teaching subjects on control systems and industrial automation at technical universities. The paper presents proven methods on how to organize and moderate an educational process with an active participation of students during theoretical and practical work over the web. Such an approach does not limit the number nor the location of participants-students, teachers or consultant workers. The international and multicultural dimension is also the most challenging one, since the cooperation can be worldwide. The work with students requires a continuous development of syllabi, since the evolution and adaptation to the changing world is continuous too. The system is open to all the participants and the nature of all of the parties involved in the system can be corporate, scientific, social or educational. The software tools used for the area of supervisory control are presented in the paper, as well as ways on how to promote this work so that new members can use it.
\end{abstract}

\section{Introduction}

New theoretical knowledge, software, hardware and communication technology bring the challenge of improving existing, and designing new, ways of interaction between students and teachers of technical universities and improving the methodology for teaching models and their implementations for various enterprises where the future graduates will go.

Please use the following format when citing this chapter:

Landryová, L., Zolotová, I., Bakoš, M., 2006, in International Federation for Information Processing, Volume 210, Education for the $21^{\text {st }}$ Century-Impact of ICT and Digital Resources, eds. D. Kumar, and Turner J., (Boston: Springer), pp. $351-355$. 
Although the syllabi at the universities may differ, the technical means, software and hardware tools, and the experience from working with them are becoming a common basis for work. The idea of sharing experiences over the web is not new, so it is just a matter of finding those common means and methods and developing cooperation, which can last long and from which everyone can benefit.

\section{Physical models of technological processes}

The physical models represent a concrete implementation environment, which includes hardware, software and communication subsystems. Their task is to help the students to learn the fundamentals of automation, logical functions, continuous and discrete control and information technologies implemented within control.

Table 4. Examples of Physical Models Used in Laboratories

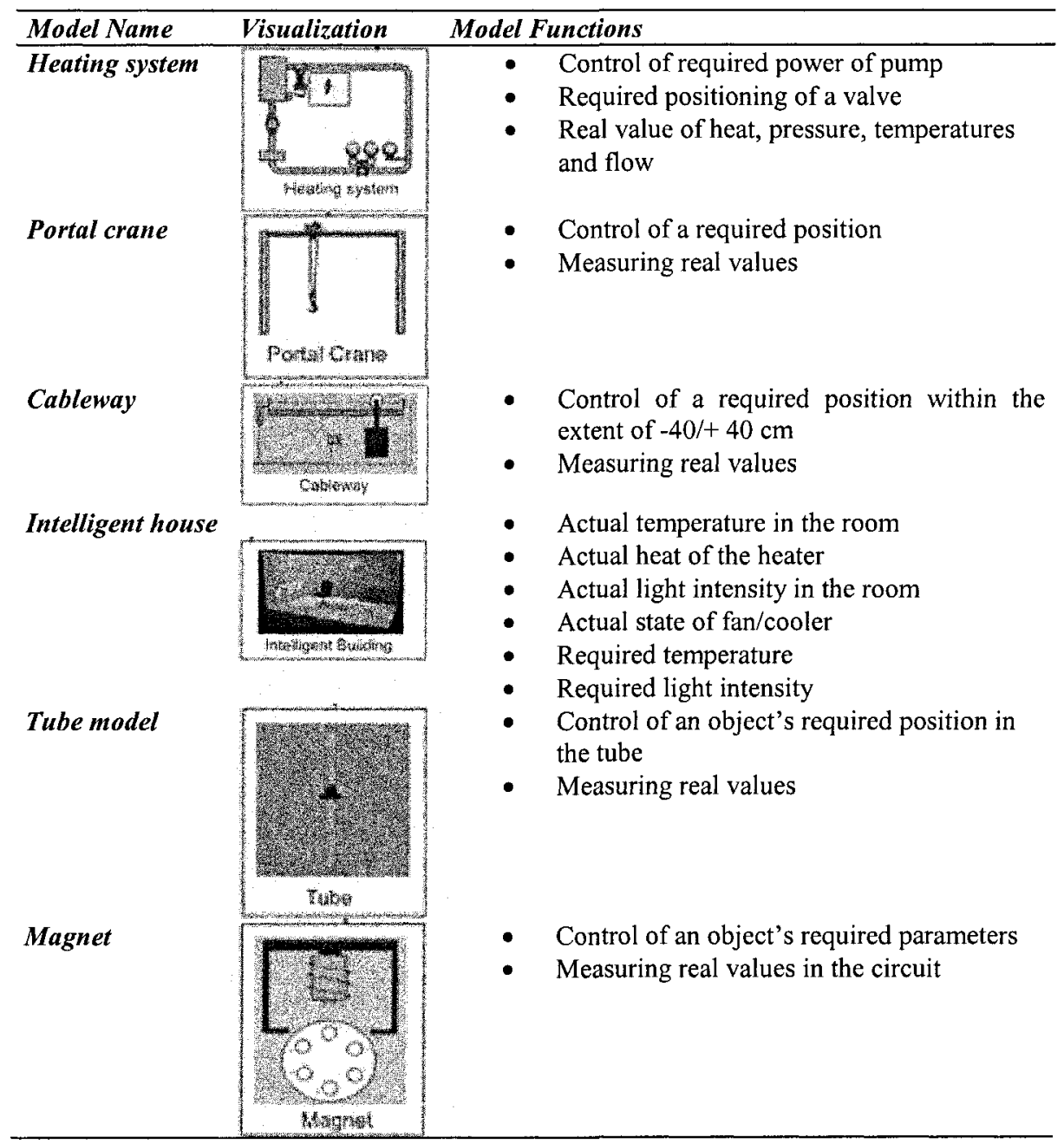


These physical models were built and developed over a period of time, during which their functionalities have proven the necessity of being used as demonstration tasks.

With the new technologies being implemented into the university laboratories it is now possible to create and maintain a virtual world based on a network of connected physical models, each of them with different communication protocols, database systems, control methods, and software tools implemented. Table 1 shows an overview of physical models.

\section{Information industrial portal}

A portal is browser-based and has the commonality of features that make it an attractive option for providing a view into a plant and its processes. These vital features include:

- information aggregation capabilities,

- common navigation tools,

- security reliability,

- extensibility and scalability of the entire system,

- multi-lingual capabilities,

- ease of use, user friendly environment,

- lower cost for providing real time information,

- views into multiple sites.

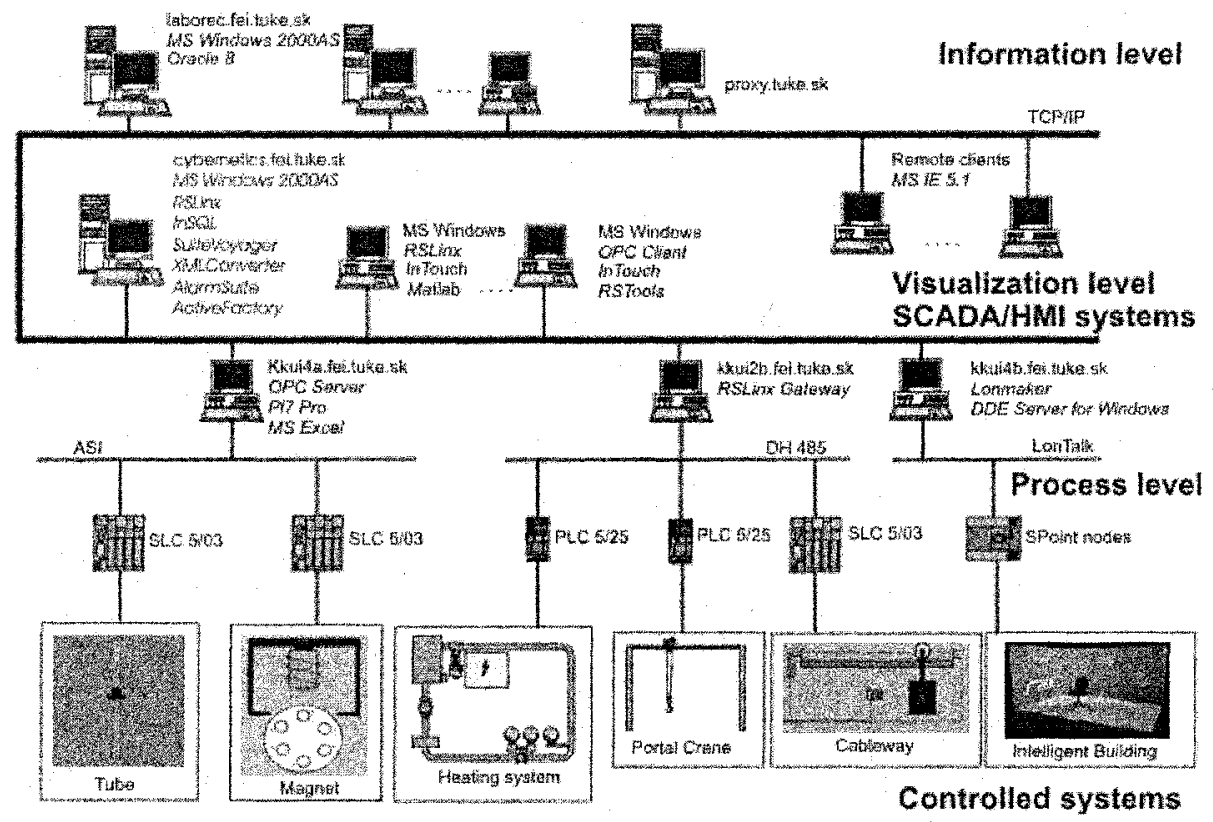

Figure 6. A part of the system architecture. 
Figure 1 shows the hierarchical levels representing different tasks. The Control Systems level consists of physical models being physically constructed, built and configured for communication with a higher level, where they were programmed with either logical functions for two-state control or PID algorithms.

At the Process level the main task is the control and data acquisition, and transfers of data in both directions. The communication protocols and drivers must be set based on the hardware and software tools available at each laboratory.

The Visualisation level represents a very comfortable task for the development of an application design in the SCADA/HMI (Supervisory Control and Data Acquisition/Human Machine Interface) system environment. This work requires skills in designing visualization screens and knowledge of up-to-date technology.

Some of the parts of logical and physical models have been published in [1].

\section{Results achieved}

The portal <http://cybernetics.fei.tuke.sk/SuiteVoyager > provides its users, students and teachers with:

- Theoretical knowledge represented by a tutorial on the introduction to theories, a model description, a user manual of supervisory control, and exercises with physical models.

- Practical experience provided from monitoring and supervisory control with the visualization of scenes, alarms, trends of the variables provided by the designed applications.

Each controlled model has its designed consistent view in the following structure:

- tutorial:

- start - short description, photo or web cam, panorama.

- model description - logical and physical models of the whole controlled system, and its integration within the information system.

- supervisory control - the user manual for HMI with demonstration video.

- exercise with the functionalities of a model.

- visualization - multi views, with control panels for supervisory control, indicators, alarm status and acknowledgment, live video via web cam.

- alarms - multi views, with current, historical alarms.

- history - adjustable history trend, portal history trend (30 min), portal history trend ( $8 \mathrm{hrs}$ ).

A model of TVM, to monitor and control a laboratory model of an air-heating aggregate, was created. This application was created with the SCADA/HMI program InTouch 8. For communication between the model and application in InTouch, an ActiveX component was created. The TVM application was translated into XML and is available on internet information portal SuiteVoyager also. Users with access rights may watch measured variables and control the model just with the help of the internet browser. 
From an education point of view the application provides:

- regulation of temperature by action intervention of the fan.

- list of prepared schemes of control.

- window enabling settings of communication.

\section{Conclusion}

The concept of a virtual laboratory is not new. Virtual labs use software simulation of physical devices and systems. Remote labs offer remote access to real physical laboratory systems, e.g., to equipment, instruments and educational models, with the goal of their monitoring and control. The educational aspects are as important as the technological ones.

The remote labs provide students with the possibility of monitoring the current situation and historical data with the help of only a basic web browser. It is available from the address http://cybernetics.fei.tuke.sk/CyberVirtLab, and the system will gradually develop. It suits the purpose of supporting learning courses by the ability to share facilities of expensive instruments and equipment. This teaching method is implemented into a real industrial portal environment, which brings a further advantage: the students learn the environment, which they will work with after graduating from the university and going into industry. The industrial information portal has proven its functionality when teaching control systems with the help of the web.

\section{Acknowledgement}

The work presented in the paper is supported by the Slovak Grant Agency KEGA funded project 3/120603 and by the Czech Ministry of Education granted project 1P05LA266.

\section{Reference}

1. Zolotová, I. et al., 2002, Contribution to Models of Supervisory Control, Data Acquisition and Human Machine Interface. Acta Electrotechnica et Informatica, Vol. 2, No. 2, FEI TU Košice, Slovak Republic, pp.62-67. 